ORIGINAL ARTICLE

\title{
Mortality and cancer morbidity in a cohort of Canadian petroleum workers
}

\author{
R J Lewis, A R Schnatter, I Drummond, N Murray, F S Thompson, A M Katz, G Jorgensen, \\ M J Nicolich, D Dahlman, G Thériault
}

Occup Environ Med 2003;60:918-928

See end of article for authors' affiliations ....................

Correspondence to: DrR J Lewis, ExxonMobil Biomedical Sciences, Inc. 1545 Route 22, PO Box 971, Room LF 264, Annandale, NJ 08801 0971, USA

r.jeffrey.lewis@

exxonmobil.com

Accepted 23 June 2003

\begin{abstract}
Aims: To assess mortality and cancer morbidity in Canadian petroleum workers and explore exposureresponse relations for specific petroleum agents.

Methods: A total of 25292 employees hired between 1964 and 1994 were linked to the Canadian tumour registry and national mortality database. Exposure-response trends were assessed for hydrocarbon solvents/fuels, hydrocarbon lubricants, petroleum coke/spent catalyst, and hydrogen sulphide $\left(\mathrm{H}_{2} \mathrm{~S}\right)$.

Results: External comparison analyses (mortality and incidence) showed deficits for all causes and all malignant neoplasms combined and were consistent with expectation for most malignant and nonmalignant sites analysed. Gall bladder cancer mortality was increased among males based on four deaths, but cases had no common job assignments and the increase was focused in workers employed $<10$ years. Mesothelioma incidence was increased. Most exposure-specific analyses were compromised by small numbers. Statistically significant increases were observed for $\mathrm{H}_{2} \mathrm{~S}$ exposure and a subgroup of accidental deaths as well as for petroleum coke/spent catalyst exposure and lung cancer. While both findings have a degree of biologic plausibility, the $\mathrm{H}_{2} \mathrm{~S}$ association, which exhibited a clearer exposureresponse pattern, could be subject to unmeasured confounders. Additionally, interpretation was complicated by the high correlation between hydrocarbon and $\mathrm{H}_{2} \mathrm{~S}$ exposures. With regard to lung cancer, the analysis could not adequately control for smoking, was based on small numbers, and exhibited a tenuous exposure-response pattern.

Conclusion: The findings for mesothelioma suggest the need for continued attention to asbestos in the petroleum industry. The relation between accidental deaths and $\mathrm{H}_{2} \mathrm{~S}$ exposure deserves closer scrutiny in similarly exposed populations. Further analyses of lung cancer are underway and will be reported separately.
\end{abstract}

W recently extended mortality follow up to 1994 for a large cohort of employees working between 1964 and 1983 in diverse operating segments of a Canadian petroleum company. ${ }^{1}$ The main finding was a statistically significant increase in mesothelioma among refinery/petrochemical workers. Increases were also observed for multiple myeloma and aortic aneurysms among marketing/distribution workers, although no clear patterns by duration of employment nor time since hire were evident. Earlier studies of this cohort ${ }^{23}$ found increased malignant melanoma and multiple myeloma among upstream (jobs involving exploration and production of natural gas and crude oil) and marketing/distribution workers, respectively. These latter two findings were related to duration of employment, but not to estimated hydrocarbon exposure frequency. ${ }^{3}$

The cohort for the present study differs from previous company cohorts ${ }^{12}$ in several ways. First, the cohort is an inception cohort from 1964 onwards, a time period more relevant to modern day work environments. Second, we included only employees with relatively complete work histories and utilised a company exposure tracking system to develop semi-quantitative exposure estimates for select petroleum agents. Finally, we utilised the Canadian Cancer Data Base (CCDB), a population based tumour registry, to obtain cancer incidence data, in addition to mortality information. However, because employees were relatively young and exposures were generally low, statistical power is limited except for causes of death and diseases that may be more prevalent among younger persons and/or have shorter latencies.

\section{METHODS}

Cohort identification and definition

We identified the cohort using the company's personnel, payroll, and human resources databases, which have been in existence since 1964. The cohort included all employees first hired between 1 January 1964 and 31 December 1994 who had at least one year of employment. As such, employees from the previous cohort ${ }^{1}$ who were retired and alive as of 1964 and employees hired before 1964 were excluded $(n=16200)$. This definition eliminates potential survivor bias by excluding retirees and persons working as of 1964 . We also excluded employees having less than $90 \%$ of their total company work time assigned to similar exposure groups (defined later) $(\mathrm{n}=325)$. The final cohort comprised

\footnotetext{
Abbreviations: CCDB, Canadian Cancer Database; $C E$, cumulative exposure; CMDB, Canadian Mortality Data Base; ETHIS, exposure tracking and health information system; GEP, good epidemiology practice; HRMS, human resources management system; IARC, International Agency for Research on Cancer; ICD, International Classification of Diseases; LHC, lymphohaematopoietic cancer; NDI National Death Index; OCMAP, Occupational Cohort Mortality Analysis Program; OEL, occupational exposure limit; $\mathrm{PAH}$, polycyclic aromatic hydrocarbons; RR, relative rate; SC, Statistics Canada; SEG, similar exposure group; SIR, standardised incidence ratios; SMR, standardised mortality ratios
} 
Main messages

- Results indicate a favourable overall health profile, with deaths due to all causes and all cancers less than expected.

- Cancer incidence findings were similar, although mesothelioma was increased in long duration/latency employees.

- Exposure-response analyses suggested a trend for cumulative $\mathrm{H}_{2} \mathrm{~S}$ exposure and transportation accidents. However, due to study limitations (for example, unmeasured confounders) and a lack of similar studies with which to compare results, findings need to be verified in other similarly exposed populations.

- Lung cancer risk was increased among workers in the highest cumulative exposure category for petroleum coke/spent catalyst. However, there was inadequate control for smoking, small numbers, and the exposureresponse pattern was tenuous. Additional larger studies with improved data on smoking and exposure may help clarify the findings and the broader question of lung cancer risk in petroleum workers.

$\mathrm{n}=25292$ employees, of which $\mathrm{n}=18360$ were also included in the previous mortality update. ${ }^{1}$ Thus, the cohort is smaller and younger than previous cohorts. ${ }^{12}$

\section{Work history summarisation and confounding factor data}

The work history variables included work site, department, function (within department), and job title. All changes in these variables were tracked, along with the effective date of change. Gaps in employment due to factors such as layoffs were accounted for.

Data on history of tobacco use (cigarettes, cigars, and pipes) were obtained from computerised medical files. These data were only sufficient to classify workers as having ever/ never (or unknown) smoked. Information on smoking history was missing for $57 \%(n=14375)$ of the cohort. A comparison of employees with and without smoking information classified by demographic variables (for example, age, time period) indicated differences of approximately $10 \%$ or less within the classes.

\section{Data quality assurance}

All aspects of the study were conducted according to good epidemiology practice (GEP) guidelines. ${ }^{4}$ We performed quality checks on fields abstracted from the payroll and personnel systems to ensure that dates were valid, complete, and formed logical progressions; this included out of range checks as well as consistency checks between fields.

\section{Exposure assessment}

The exposure estimating methods are described in detail elsewhere. ${ }^{5}$ In 1987, the company implemented an exposure tracking and health information system (ETHIS). The ETHIS groups employees by similar exposure group (SEG) codes using location, department, function, and dates. The SEG code describes potential exposures among employees who perform similar work and have similar exposure potential. The SEG codes include estimates of exposure that were developed by an industrial hygienist through interviews, chemical inventories, IH measurements (where available), and their knowledge of the workplace. In many instances, data from similar units or worksites were used.
Each SEG code is linked to a substance (that is, an ETHIS agent). Each ETHIS agent contains a record indicating estimates of the exposure concentration (based on one of four centile categories of the occupational exposure limit $(\mathrm{OEL})$ ) and exposure frequency (as fraction of a year exposed). Since the work histories used in this study extended back to 1964 and the ETHIS system started in 1987, unassigned work history lines were linked to new or existing SEGs by two industrial hygienists familiar with the work site. ${ }^{5}$

\section{Selection and definition of study agents}

We developed the following criteria to select agents for study: the potential hazard of the substance, the ability to generate new hypotheses, the lack of good exposure-response information in human populations, the existence of high quality exposure measurements or estimates, prevalence of exposure, and related factors. Application of these criteria resulted in the selection of five study agents: hydrocarbon solvents/fuels, hydrocarbon lubricants, petroleum coke, hydrogen sulphide $\left(\mathrm{H}_{2} \mathrm{~S}\right)$, and a general indicator for exposure to operations.

Hydrocarbon solvents/fuels included saturated, alicyclic, aromatic, and olefinic hydrocarbons with carbon numbers from C5 to $\mathrm{C} 16$, boiling points from $35^{\circ}$ to $338^{\circ} \mathrm{C}$. Hydrocarbon lubricants included base stock oils and finished products obtained by blending additives into these oils. Hydrocarbon lubricants include some "mildly refined" components such as middle distillate solvent extract. Examples of hydrocarbon lubricants include engine, gear and hydraulic oils, electrical and heat transfer oils, automotive and industrial greases, and speciality lubricants. Petroleum coke is associated with a number of refining processes, including coking, catalytic cracking, hydrocracking, reforming, and hydroforming. We defined exposure to include both exposure to fine dust particles of petroleum coke as a by-product, as well as exposure to catalyst dusts containing small quantities of petroleum coke (hereafter referred to as petroleum coke/spent catalyst). Hydrogen sulphide, the only "pure" substance agent assessed, is a major component of many production or process streams and some products. We subsequently dropped the general exposure indicator, since findings were unremarkable.

\section{Exposure estimation process}

All exposure estimation was performed without knowledge of the individual's disease status. Once all SEG codes that contained one or more of the study agents were identified, exposure categories (ordinal rankings) were assigned to all work history lines having these SEG codes. Cumulative exposure (CE) was calculated as

$$
\sum_{\text {jobs }} \sum_{\text {time }} C \times F \times Y,
$$

where $C=$ concentration (based on one of four centile categories of the OEL), $F=$ frequency of exposure (fraction of year exposed), and $Y=$ number of years in job. Similar methods were used to calculate CE for those study agents comprised of multiple ETHIS agents. ${ }^{5}$

Finally, we evaluated the quality of the exposure estimates compared with industrial hygiene measurement surveys. Based on a sample of $460 \mathrm{IH}$ surveys carried out during varying time periods, there was a high level of agreement between measured and estimated exposures. ${ }^{5}$

\section{Vital status ascertainment}

All 25292 employees were submitted to the Canadian Mortality Data Base (CMDB) maintained by Statistics Canada (SC) for the years 1964 to 1994. Additional deaths 
were identified using the US National Death Index (NDI) and company death notices.

Underlying cause of death was determined by SC using the International Classification of Diseases (ICD) code, revision in effect, for deaths identified via the CMDB linkage. For all remaining deaths, underlying cause was determined by a single certified nosologist. Due to potential vinyl chloride monomer and asbestos exposure at certain locations, a certified nosologist reviewed all death certificates for selected ICD codes (see appendix of Schnatter and colleagues ${ }^{2}$ ) for any mention of angiosarcoma or mesothelioma.

\section{Cancer incidence ascertainment}

We identified incident cancers diagnosed among employees between 1969 (the cancer registry start date) and 1994 using Statistics Canada's CCDB. The CCDB's linkage procedures, which involve the use of customised rules for matching records, were used. Information obtained for all tumours identified included date of diagnosis, ICD code (9th revision), method of diagnosis, histological classification, and source of registration. Instances where employees had more than one record indicating a malignancy were reviewed by SC to determine if the records were duplicate reports or independent, multiple primary tumours. The International Agency for Research on Cancer's (IARC) rules for determining multiple primary cancers ${ }^{6}$ were followed for all malignant sites except for lymphoma and leukaemia, where the surveillance, epidemiology and end results rules were used. ${ }^{7}$ Malignant neoplasm deaths identified by the CMDB that were not identified as incident tumours by the CCDB were also counted as incident tumours.

\section{Analysis}

Diseases of a priori interest in the general surveillance analyses included causes that were increased in previous company cohorts (multiple myeloma, malignant melanoma, mesothelioma, and large intestine cancer, excluding rectum $)^{1-38}$ or in other petroleum or petrochemical industry cohorts (lymphohaematopoietic related cancers (LHC), brain and kidney cancer, and angiosarcoma).$^{9-11}$

\section{Mortality}

Employees were entered into follow up on their 365th work day. Person-years at risk were summed by five-year categories of age and time until date of death or end of study (31 December 1994), whichever was earliest. Owing to the relative completeness of the $\mathrm{CMDB}^{12}$ and the $\mathrm{NDI}^{13}$ persons of unknown vital status were assumed to be alive until the end of study. Expected deaths were calculated using a modified life table approach with the Occupational Cohort Mortality Analysis Program (OCMAP), version 2.0. ${ }^{14}$ Mesothelioma incidence rates based on SC's tumour registry were used to calculate expected deaths (see Lewis and colleagues $^{1}$ for more detail). Exact $95 \%$ confidence intervals ${ }^{15}$ were calculated for standardised mortality ratios (SMR) under the assumption that the observed numbers of deaths followed a Poisson distribution. Unless the SMR was statistically significant, we do not present SMR nor 95\% confidence intervals when both the observed and expected number of deaths are less than five to avoid presentation of less meaningful results.

\section{Cancer incidence}

Standardised incidence ratios (SIR) were calculated, with expected malignancies being based on incidence rates for the general Canadian population. We excluded seven employees from the analysis who died before $1 / 1 / 69$ (the date of the CCDB's inception).
Workers hired between 1/1/64 and 1/1/68 who had worked at least 365 days were entered into follow up on 1/1/69, while all other employees were entered into follow up on their 365 th working day. Employees were considered to be at risk until date of first tumour diagnosis after initial employment with the company, date of death, or end of study, whichever was earliest.

\section{Substance specific analyses}

We used Poisson regression techniques to evaluate the relation between selected diseases and exposure to the four study agents. We focused on outcomes that were biologically plausible for each agent, or that have been previously associated with the agent to reduce the likelihood of chance findings. Only outcomes having 10 or more exposed cases are presented to avoid presentation of less meaningful results (there were no significant findings among analyses not presented). These analyses are based on cancer incidence and non-cancer outcomes.

We used SAS $^{16}$ to obtain the maximum likelihood relative rate (RR) and 95\% confidence interval associated with cumulative exposure (CE) to each study agent relative to unexposed subjects. All regression models for malignant outcomes included categorical variables for age $(<40, \geqslant 40$ $59, \geqslant 60+)$, calendar period (1969-79, 1980-89, 1990-94), and time since hire $(<10, \geqslant 10$ years $)$. Categorisation of cumulative exposure was defined a priori based on (a) tertiles of exposure and (b) $50 \%, 75 \%$, and $90 \%$ of the exposure distribution among exposed subjects. Due to similarity of results, only tertile findings are shown.

\section{RESULTS}

\section{Characteristics of the cohort}

Table 1 presents the vital status, demographic, and employment characteristics of the cohort. This is a young cohort which, on average, was employed for less than 10 years. Only $2 \%$ of the cohort were deceased and $0.7 \%$ died of malignancies. Two per cent of the cohort had one or more incident malignancies.

\section{Mortality}

Deficits of deaths due to all causes and all malignant neoplasms were observed for both males and females (see

Table 1 Vital status, demographic, and employment characteristics of employees from a Canadian petroleum company, by gender

\begin{tabular}{|c|c|c|}
\hline \multirow[b]{2}{*}{ Characteristics } & \multicolumn{2}{|l|}{ Total cohort } \\
\hline & Males & Females \\
\hline Total & 17230 & 8062 \\
\hline Alive (\% of total)* & $16768(97.3)$ & $7953(98.6)$ \\
\hline Deceased $(\% \text { of total })^{*}$ & $462(2.7)$ & $109(1.4)$ \\
\hline $\begin{array}{l}\text { No. of persons with one or more } \\
\text { incident tumours identified } \dagger\end{array}$ & $325(1.9 \%)$ & $184(2.3 \%)$ \\
\hline Average year of entry into follow up & 1978.8 & 1978.9 \\
\hline Average age at entry into follow up & 29.1 & 27.5 \\
\hline Average year of hire & 1977.5 & 1977.8 \\
\hline $\begin{array}{l}\text { Average duration of employment } \\
\text { (years) }\end{array}$ & 8.8 & 6.4 \\
\hline $\begin{array}{l}\text { Average follow up time (since } \\
\text { hire in years) }\end{array}$ & 16.8 & 16.7 \\
\hline Total person-years & 268290 & 125522 \\
\hline \multicolumn{3}{|c|}{$\begin{array}{l}\text { *As of end of follow up ( } 31 \text { December 1994). } \\
\text { †Seven males who died before } 1 \text { January } 1969 \text { (date tumour registry } \\
\text { ascertainment began) were excluded from cancer incidence analysis } \\
\text { cohort. Additionally, six males and two females who had a death } \\
\text { certificate indicating a malignant neoplasm death but who were not } \\
\text { identified by the tumour registry as incident cancers were included as } \\
\text { tumour cases. }\end{array}$} \\
\hline
\end{tabular}


Table 2 Mortality results* for employees of a Canadian petroleum company, by gender, 1964-94

\begin{tabular}{|c|c|c|c|c|}
\hline \multirow[b]{2}{*}{ Cause of death (ICD codes, 8th revision) } & \multicolumn{2}{|l|}{ Males } & \multicolumn{2}{|l|}{ Females } \\
\hline & O/E & SMR $(95 \% \mathrm{Cl})$ & O/E & SMR $(95 \% \mathrm{Cl})$ \\
\hline All causes of death (000-999) & $462 / 711.86 \dagger$ & $0.65(0.59$ to 0.71$)$ & $109 / 158.91 \dagger$ & $0.69(0.56$ to 0.83$)$ \\
\hline Infective \& parasitic diseases (000-136) & $3 / 23.59+$ & 0.13 (0.03 to 0.37$)$ & $0 / 1.90$ & - \\
\hline Malignant neoplasms (140-209) & $123 / 163.42 \dagger$ & $0.75(0.63$ to 0.90$)$ & $56 / 65.80$ & $0.85(0.64$ to 1.10$)$ \\
\hline Oesophagus (150) & $2 / 4.19$ & - & $1 / 0.42$ & - \\
\hline Stomach (151) & $4 / 7.46$ & $0.54(0.15$ to 1.37$)$ & $0 / 1.67$ & - \\
\hline Large intestine (except rectum) (153) & $14 / 14.07$ & $1.00(0.54$ to 1.67$)$ & $2 / 4.99$ & $0.40(0.05$ to 1.45$)$ \\
\hline Rectum and rectosigmoid junction (154) & $4 / 4.62$ & - & $1 / 1.10$ & - \\
\hline Liver (155.0) & $0 / 1.98$ & - & $0 / 0.31$ & - \\
\hline Gall bladder (156) & $4 / 0.94 \dagger$ & $4.28(1.17$ to 10.95$)$ & $0 / 0.52$ & - \\
\hline Pancreas (157) & $4 / 7.81$ & $0.51(0.14$ to 1.31$)$ & $1 / 2.07$ & - \\
\hline Nose \& sinuses (160) & $2 / 0.35$ & - & $0 / 0.7$ & - \\
\hline Bronchus \& lung (162.1) & $35 / 49.81 \dagger$ & 0.70 (0.49 to 0.98$)$ & $7 / 10.85$ & $0.64(0.26$ to 1.33$)$ \\
\hline Pleura (163.0) & $0 / 0.48$ & - & $0 / 0.07$ & - \\
\hline Bone (170) & $0 / 0.95$ & - & $0 / 0.25$ & - \\
\hline Malignant melanoma (172) & $3 / 4.52$ & - & $1 / 1.37$ & - \\
\hline Breast (174) & $0 / 0.18$ & - & $20 / 18.45$ & $1.08(0.66$ to 1.67$)$ \\
\hline Cervix uteri (180) & & & $3 / 2.97$ & - \\
\hline Corpus uteri (182.0) & & & $0 / 0.51$ & - \\
\hline Ovary, fallopian tube, \& broad ligaments (183) & & & $7 / 4.03$ & $1.74(0.70$ to 3.58$)$ \\
\hline Prostate (185) & $1 / 4.61$ & - & & \\
\hline Testis (186) & $3 / 1.61$ & - & & \\
\hline Bladder (188) & $2 / 2.30$ & - & $0 / 0.32$ & - \\
\hline Kidney (189.0-189.2) & $3 / 4.79$ & - & $1 / 0.92$ & - \\
\hline Brain (malignant) (191) & $11 / 9.29$ & $1.18(0.59$ to 2.12$)$ & $2 / 2.60$ & - \\
\hline Malignant CNS tumours (excluding brain) (192) & $1 / 0.44$ & - & $1 / 0.14$ & - \\
\hline $\begin{array}{l}\text { All nervous system neoplasms (malignant, } \\
\text { benign \& unspec.) }(191,192,225,238)\end{array}$ & $12 / 11.02$ & $1.09(0.56$ to 1.90$)$ & $3 / 3.19$ & - \\
\hline Reticulum cell sarcoma (200.0) & $0 / 0.62$ & - & $0 / 0.16$ & - \\
\hline Lymphosarcoma (200.1) & $0 / 0.73$ & - & $1 / 0.18$ & - \\
\hline Hodgkin's disease (201) & $3 / 2.50$ & - & $1 / 0.63$ & - \\
\hline Non-Hodgkin's lymphoma $(200,202.0,202.2)$ & $5 / 7.45$ & $0.67(0.22$ to 1.57$)$ & $3 / 1.94$ & - \\
\hline Other lymphoid neoplasms $(202.1,202.9)$ & $1 / 0.45$ & - & $0 / 0.11$ & - \\
\hline Multiple myeloma (203) & $1 / 2.16$ & - & $0 / 0.63$ & - \\
\hline Leukaemias (204-207) & $7 / 8.18$ & $0.86(0.34$ to 1.76$)$ & $2 / 2.63$ & - \\
\hline Acute non-lymphocytic leukaemia $(205.0,206.0,207.2)$ & $4 / 2.48$ & - & $0 / 0.95$ & - \\
\hline Acute lymphoid leukaemia (204.0) & $1 / 0.90$ & - & $0 / 0.28$ & - \\
\hline Chronic lymphoid leukaemia (204.1) & $1 / 0.75$ & - & $0 / 0.12$ & - \\
\hline Acute myeloid (myelomonocytic) leukaemia (205.0) & $3 / 2.37$ & - & $0 / 0.90$ & - \\
\hline Chronic myeloid leukaemia (205.1) & $1 / 1.46$ & - & $0 / 0.43$ & - \\
\hline $\begin{array}{l}\text { Other leukaemia }(204.9,205.9,206.1,206.9 \text {, } \\
\text { 207.0-207.1, 207.9) }\end{array}$ & $0 / 2.57$ & - & $2 / 0.84$ & - \\
\hline Benign neoplasms (210-228) & $0 / 0.54$ & - & $0 / 0.28$ & - \\
\hline Endocrine, nutritional, \& metabolic diseases (240-279) & $14 / 14.68$ & $0.95(0.52$ to 1.60$)$ & $3 / 3.83$ & - \\
\hline Diabetes mellitus (250) & $3 / 9.75 \dagger$ & $0.31(0.06$ to 0.90$)$ & $3 / 2.62$ & - \\
\hline Aids (275.1) & $9 / 20.00 \dagger$ & $0.45(0.21$ to 0.85$)$ & $0 / 0.50$ & - \\
\hline Blood diseases (280-289) & $0 / 1.47$ & - & $0 / 0.60$ & - \\
\hline Amyotrophic lateral sclerosis (348.0) & $1 / 1.58$ & - & $1 / 0.43$ & - \\
\hline Circulatory diseases $(390-458)$ & $112 / 183.20 \dagger$ & $0.61(0.50$ to 0.74$)$ & $22 / 28.46$ & $0.77(0.48$ to 1.17$)$ \\
\hline Acute myocardial infarction (410) & $47 / 85.75 \dagger$ & $0.55(0.40$ to 0.73$)$ & $4 / 8.58$ & $0.47(0.13$ to 1.19$)$ \\
\hline Cerebrovasular disease $(430-438)$ & $13 / 20.76$ & $0.63(0.33$ to 1.07$)$ & $8 / 7.59$ & $1.05(0.46$ to 2.08$)$ \\
\hline Diseases of arteries, arterioles, \& capillaries (440-448) & $5 / 6.68$ & $0.75(0.24$ to 1.75$)$ & $2 / 1.26$ & - \\
\hline Aortic aneurysm (441) & $4 / 3.84$ & - & $1 / 0.47$ & - \\
\hline Respiratory diseases (460-519) & $15 / 22.03$ & $0.68(0.38$ to 1.12$)$ & $2 / 5.90$ & $0.34(0.04$ to 1.22$)$ \\
\hline Chronic obstructive pulmonary disease (519.3) & $2 / 5.25$ & $0.38(0.05$ to 1.38$)$ & $1 / 1.08$ & - \\
\hline Digestive diseases $(520-577)$ & $21 / 31.66$ & $0.66(0.41$ to 1.01$)$ & $4 / 6.69$ & $0.60(0.16$ to 1.53$)$ \\
\hline Cirrhosis of liver (571) & $13 / 20.05$ & $0.65(0.35$ to 1.11$)$ & $3 / 3.52$ & - \\
\hline Diseases of pancreas (577) & $2 / 1.77$ & - & $0 / 0.30$ & - \\
\hline Genitourinary diseases (580-629) & $3 / 4.46$ & - & $0 / 1.52$ & - \\
\hline Kidney diseases (580-593) & $3 / 4.08$ & - & $0 / 1.33$ & - \\
\hline Accidents, poisonings, \& violence (E800-E999) & $153 / 222.20+$ & 0.69 (0.58 to 0.81$)$ & $13 / 31.67 \dagger$ & $0.41(0.22$ to 0.70$)$ \\
\hline Suicide (E950-E959) & $44 / 69.84 \dagger$ & $0.63(0.46$ to 0.85$)$ & $8 / 9.93$ & $0.81(0.35$ to 1.59$)$ \\
\hline
\end{tabular}

*Expected deaths based on Canadian general population mortality rates.

+95\% Cl does not include 1.00 .

$\mathrm{O} / \mathrm{E}$, observed/expected.

table 2). Mortality was notably less than or consistent with expected for causes more prevalent among younger age groups (for example, AIDS, accidents, poisonings, and violence) and for cancers with potentially shorter latencies (for example, LHC). The only statistically significant increase observed was for gall bladder cancer among males (SMR 4.3;
95\% CI 1.2 to 11.0 ), based on only four cases. A review of work histories for the four gall bladder cancer deaths and the one incident only case indicated no consistent pattern in job type. There were limited numbers of female deaths for most causes, although ovarian related cancers were non-significantly increased (SMR 1.7). 
Only one death due to mesothelioma was observed versus 1.3 expected (data not shown). No deaths due to angiosarcoma were identified.

\section{Cancer incidence}

There were 501 employees identified by the CCDB as having one or more malignancy. Additionally, there were eight employees identified by the CMDB as having died from a malignant neoplasm who were not identified as incident cases in the tumour registry. The eight employees showed no clustering by year or cause of death. All analyses are based on first diagnosis in the 509 subjects.

SIRs were generally slightly higher compared with SMRs, although both males and females had less than expected incidence of all malignancies combined (see table 3). Males experienced a non-significant increase in chronic lymphoid leukaemia (SIR 1.81) and gall bladder cancer (SIR 2.84). All six male chronic lymphoid leukaemia cases worked primarily in office related jobs.

There were four mesothelioma cases identified among males versus 1.32 expected. Work histories indicated two of the four cases were long term workers having potential asbestos exposure. There was one angiosarcoma identified in a short term employee who worked in a job where no vinyl chloride exposures would have occurred (data not shown).

Table 4 shows analyses by tenure for males with $\geqslant 10$ years since first employment for select cancers. This analysis was hampered by small numbers. There was no pattern of increasing risk with increasing tenure evident for all cancers combined or for lung cancer. However, SIRs increased with increasing duration of employment for large intestine cancer. Small numbers for females precluded analyses for most sites (data not shown), although females showed no increasing trends with tenure for cancers of the large intestine or breast, or ovarian related cancers.

\section{Substance specific results}

Table 5 shows that concentration estimates were quite low (for example, $75 \%$ of exposures were $1 \mathrm{ppm}$ or less for $\mathrm{H}_{2} \mathrm{~S}$ ). The correlation between petroleum coke/spent catalyst and the other three agents was generally low (Spearman rank correlation coefficients of 0.2 to 0.4 ) but was relatively high among the other three agents (Spearman rank correlation coefficients of 0.5 to 0.7 ).

\section{Hydrocarbon solvents/fuels}

Table 6 shows that there was little evidence of increased risk among employees exposed to hydrocarbon solvents/fuels, although many results were based on small numbers. Risk of brain cancer was non-significantly increased among the middle (RR 2.7) and upper exposure categories (RR 2.0).

\section{Hydrocarbon lubricants}

Table 7 shows a possibly weak increasing trend for brain cancer and hydrocarbon lubricant exposure, although none of the individual RR were statistically significant. For cancers

Table 3 Cancer incidence results* for employees of a Canadian petroleum company, by gender, 1969-94

\begin{tabular}{|c|c|c|c|c|}
\hline \multirow{2}{*}{$\begin{array}{l}\text { Type of malignancy } \\
\text { (ICD codes, 9th revision) }\end{array}$} & \multicolumn{2}{|l|}{ Males } & \multicolumn{2}{|l|}{ Females } \\
\hline & $\mathrm{O} / \mathrm{E}$ & $\operatorname{SIR}(95 \% \mathrm{CI})$ & $\mathrm{O} / \mathrm{E}$ & $\operatorname{SIR}(95 \% \mathrm{CI})$ \\
\hline Total malignant neoplasms (140-208) & $325 / 379.11 \dagger$ & $0.86(0.77$ to 0.96$)$ & $184 / 212.16+$ & $0.87(0.75$ to 1.00$)$ \\
\hline Oesophagus (150) & $3 / 4.33$ & - & $0 / 0.48$ & - \\
\hline Stomach (151) & $6 / 12.09$ & 0.50 (0.18 to 1.08$)$ & $0 / 2.59$ & - \\
\hline Large intestine (except rectum) $(153,159.0)$ & $33 / 27.84$ & $1.18(0.82$ to 1.66$)$ & $12 / 10.72$ & $1.12(0.58$ to 1.96$)$ \\
\hline Rectum and rectosigmoid junction (154) & $15 / 18.74$ & $0.80(0.45$ to 1.32$)$ & $4 / 5.31$ & $0.75(0.20$ to 1.93$)$ \\
\hline Liver (155.0) & $2 / 3.14$ & - & $0 / 0.46$ & - \\
\hline Gall bladder (156) & $5 / 1.76$ & $2.84(0.92$ to 6.62$)$ & $0 / 0.87$ & - \\
\hline Pancreas (157) & $5 / 8.42$ & $0.59(0.19$ to 1.39$)$ & $0 / 2.27$ & - \\
\hline Nose \& sinuses $(160)$ & $2 / 1.19$ & - & $1 / 0.31$ & - \\
\hline Bronchus \& lung (162.2-162.5, 162.8-162.9) & $44 / 63.98 \dagger$ & 0.69 (0.50 to 0.92$)$ & $9 / 15.29$ & $0.59(0.27$ to 1.12$)$ \\
\hline Pleura (163) & $3 / 1.27$ & - & $0 / 0.14$ & - \\
\hline Bone (170) & $4 / 2.30$ & - & $0 / 0.81$ & - \\
\hline Malignant melanoma (172) & $26 / 20.84$ & 1.25 (0.82 to 1.83$)$ & $16 / 10.97$ & $1.46(0.83$ to 2.37$)$ \\
\hline Breast (174-175) & $0 / 0.73$ & - & $76 / 74.45$ & $1.02(0.80$ to 1.28$)$ \\
\hline Cervix uteri (180) & & & $7 / 16.81 \dagger$ & $0.42(0.17$ to 0.86$)$ \\
\hline Corpus uteri (182) & & & $3 / 9.81 \dagger$ & 0.31 (0.06 to 0.89$)$ \\
\hline Ovary, fallopian tube, \& broad ligaments (183) & & & $15 / 10.75$ & $1.40(0.78$ to 2.30$)$ \\
\hline Prostate (185) & $20 / 30.05$ & $0.67(0.41$ to 1.03$)$ & & \\
\hline Testis (186) & $14 / 17.10$ & $0.82(0.45$ to 1.37$)$ & & \\
\hline Bladder (188) & $18 / 19.52$ & $0.92(0.55$ to 1.46$)$ & $2 / 2.75$ & - \\
\hline Kidney (189.0-189.2) & $15 / 15.01$ & $1.00(0.56$ to 1.65$)$ & $2 / 3.49$ & - \\
\hline Brain (malignant) (191) & $16 / 14.04$ & $1.14(0.65$ to 1.85$)$ & $5 / 4.14$ & $1.21(0.40$ to 2.82$)$ \\
\hline Malignant CNS (excluding brain) (192.0-192.9) & $0 / 1.08$ & - & $1 / 0.45$ & - \\
\hline Reticulum cell sarcoma (200.0) & $6 / 4.86$ & $1.24(0.45$ to 2.69$)$ & $1 / 1.41$ & - \\
\hline Lymphosarcoma (200.1) & $4 / 3.52$ & - & $2 / 1.00$ & - \\
\hline Hodgkin's disease (201) & $11 / 10.46$ & 1.05 (0.52 to 1.88$)$ & $3 / 3.34$ & - \\
\hline Non-Hodgkin's lymphoma (200.0-200.2, 202.0, 202.8) & $20 / 20.57$ & $0.97(0.59$ to 1.50$)$ & $7 / 5.87$ & $1.19(0.48$ to 2.46$)$ \\
\hline Other lymphoid neoplasms $(200.8,202.1,202.9)$ & $4 / 3.61$ & - & $0 / 0.96$ & - \\
\hline Multiple myeloma (203.0) & $3 / 3.66$ & - & $0 / 1.09$ & - \\
\hline Leukaemias (204-206, 207.0, 207.2, 207.8, 208) & $13 / 13.49$ & $0.96(0.51$ to 1.65$)$ & $3 / 4.23$ & - \\
\hline Acute non-lymphocytic leukaemia $(205.0,206.0,207.0)$ & $4 / 4.13$ & - & $2 / 1.66$ & - \\
\hline Acute lymphoid leukaemia (204.0) & $2 / 1.42$ & - & $0 / 0.42$ & - \\
\hline Chronic lymphoid leukaemia (204.1) & $6 / 3.32$ & $1.81(0.66$ to 3.94$)$ & $0 / 0.66$ & - \\
\hline Acute myeloid (myelomonocytic) leukaemia (205.0) & $3 / 3.79$ & - & $2 / 1.54$ & - \\
\hline Chronic myeloid leukaemia (205.1) & $0 / 2.84$ & - & $1 / 0.86$ & - \\
\hline $\begin{array}{l}\text { Other leukaemias (204.2-204.9, 205.2-205.9, 206.1-206.9, } \\
\text { 207.2-207.8, 208.0-208.9) }\end{array}$ & $1 / 1.67$ & - & $0 / 0.59$ & - \\
\hline
\end{tabular}

*Expected malignancies based on Canadian general population cancer incidence rates. †95* $\mathrm{Cl}$ does not include 1.00 .

$\mathrm{O} / \mathrm{E}$, observed/expected. 
Table 4 Cancer incidence* by duration of employment among male employees with at least 10 years since hire, 1969-94

\begin{tabular}{|c|c|c|c|}
\hline \multirow[b]{2}{*}{$\begin{array}{l}\text { Type of malignancy } \\
\text { (ICD codes, 9th revision) }\end{array}$} & \multirow{2}{*}{$\begin{array}{l}<10 \text { years employment } \\
\text { O/E } \\
\text { SIR }(95 \% \mathrm{Cl})\end{array}$} & \multirow{2}{*}{$\begin{array}{l}\geqslant 10-<20 \text { years } \\
\text { employment } \\
\text { O/E } \\
\text { SIR }(95 \% \mathrm{CI})\end{array}$} & \multirow{2}{*}{$\begin{array}{l}\geqslant 20 \text { years employment } \\
\mathrm{O} / \mathrm{E} \\
\text { SIR }(95 \% \mathrm{Cl})\end{array}$} \\
\hline & & & \\
\hline Malignant neoplasms (140-208) & $\begin{array}{l}120 / 146.25 \\
0.82(0.68 \text { to } 0.98) \dagger\end{array}$ & $\begin{array}{l}97 / 93.97 \\
1.03(0.84 \text { to } 1.26)\end{array}$ & $\begin{array}{l}26 / 30.75 \\
0.85(0.55 \text { to } 1.24)\end{array}$ \\
\hline Stomach (151) & $\begin{array}{l}3 / 4.83 \\
-\end{array}$ & $2 / 3.06$ & $0 / 1.01$ \\
\hline Large intestine (except rectum) $(153,159.0)$ & $\begin{array}{l}7 / 11.40 \\
0.61(0.25 \text { to } 1.26)\end{array}$ & $\begin{array}{l}10 / 7.13 \\
1.40(0.67 \text { to } 2.58)\end{array}$ & $\begin{array}{l}6 / 2.50 \\
2.40(0.88 \text { to } 5.21)\end{array}$ \\
\hline Rectum and rectosigmoid junction (154) & $\begin{array}{l}5 / 7.69 \\
0.65(0.21 \text { to } 1.52)\end{array}$ & $\begin{array}{l}3 / 4.84 \\
-\end{array}$ & $1 / 1.77$ \\
\hline Liver (155.0) & $1 / 1.27$ & $\begin{array}{l}0 / 0.82 \\
-\end{array}$ & $\begin{array}{l}0 / 0.28 \\
-\end{array}$ \\
\hline Gall bladder (156) & $\begin{array}{l}3 / 0.73 \\
-\end{array}$ & $\begin{array}{l}0 / 0.45 \\
-\end{array}$ & $\begin{array}{l}0 / 0.16 \\
-\end{array}$ \\
\hline Pancreas (157) & $\begin{array}{l}3 / 3.46 \\
-\end{array}$ & $\begin{array}{l}1 / 2.17 \\
-\end{array}$ & $\begin{array}{l}1 / 0.77 \\
-\end{array}$ \\
\hline Bronchus \& lung (162.2-162.5, 162.8-162.9) & $\begin{array}{l}21 / 26.74 \\
0.78(0.49 \text { to } 1.20)\end{array}$ & $\begin{array}{l}12 / 16.90 \\
0.71(0.37 \text { to } 1.24)\end{array}$ & $\begin{array}{l}3 / 6.19 \\
0.48(0.10 \text { to } 1.42)\end{array}$ \\
\hline Pleura (163) & $0 / 0.54$ & $\begin{array}{l}1 / 0.33 \\
-\end{array}$ & $2 / 0.13$ \\
\hline Malignant melanoma (172) & $\begin{array}{l}9 / 7.43 \\
1.21(0.55 \text { to } 2.30)\end{array}$ & $\begin{array}{l}11 / 4.95 \\
2.22(1.11 \text { to } 3.97)\end{array}$ & $2 / 1.36$ \\
\hline Prostate (185) & $\begin{array}{l}8 / 13.79 \\
0.58(0.25 \text { to } 1.14)\end{array}$ & $\begin{array}{l}6 / 8.21 \\
0.73(0.27 \text { to } 1.59)\end{array}$ & $\begin{array}{l}0 / 3.11 \\
-\end{array}$ \\
\hline Bladder (188) & 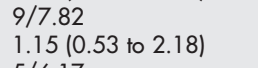 & $\begin{array}{l}5 / 4.96 \\
1.01(0.33 \text { to } 2.35)\end{array}$ & $1 / 1.62$ \\
\hline Kidney (189.0-189.2) & $\begin{array}{l}5 / 6.17 \\
0.81(0.26 \text { to } 1.89)\end{array}$ & 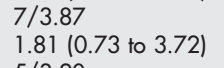 & $1 / 1.43$ \\
\hline Brain (malignant) (191) & $\begin{array}{l}7 / 4.73 \\
1.48(0.60 \text { to } 3.05)\end{array}$ & $\begin{array}{l}5 / 3.20 \\
1.56(0.51 \text { to } 3.65)\end{array}$ & $2 / 0.85$ \\
\hline Malignant CNS (excluding brain) (192.0-192.9) & $0 / 0.32$ & $\begin{array}{l}0 / 0.22 \\
-\end{array}$ & $\begin{array}{l}0 / 0.05 \\
-\end{array}$ \\
\hline Reticulum cell sarcoma (200.0) & $\begin{array}{l}4 / 1.75 \\
-\end{array}$ & $\begin{array}{l}0 / 1.18 \\
-\end{array}$ & $\begin{array}{l}1 / 0.34 \\
-\end{array}$ \\
\hline Lymphosarcoma (200.1) & $1 / 1.20$ & $\begin{array}{l}1 / 0.80 \\
-\end{array}$ & $1 / 0.22$ \\
\hline Hodgkin's disease (201) & $\begin{array}{l}3 / 2.53 \\
-\end{array}$ & $\begin{array}{l}2 / 1.83 \\
-\end{array}$ & $\begin{array}{l}0 / 0.30 \\
-\end{array}$ \\
\hline Non-Hodgkin's lymphoma $(200.0-200.2,202.0,202.8)$ & $\begin{array}{l}8 / 7.68 \\
1.04(0.45 \text { to } 2.05)\end{array}$ & $\begin{array}{l}5 / 5.07 \\
0.99(0.32 \text { to } 2.30)\end{array}$ & $3 / 1.53$ \\
\hline Other lymphoid neoplasms $(200.8,202.1,202.9)$ & $1 / 1.37$ & $3 / 0.90$ & $0 / 0.28$ \\
\hline Multiple myeloma (203.0) & $\begin{array}{l}0 / 1.54 \\
-\end{array}$ & $\begin{array}{l}3 / 0.95 \\
-\end{array}$ & $\begin{array}{l}0 / 0.35 \\
-\end{array}$ \\
\hline Leukaemias (204-206, 207.0, 207.2, 207.8, 208) & $4 / 4.74$ & $4 / 3.08$ & $0 / 0.90$ \\
\hline Acute non-lymphocytic leukaemia $(205.0,206.0,207.0)$ & $1 / 1.38$ & $1 / 0.91$ & $0 / 0.25$ \\
\hline Acute lymphoid leukaemia (204.0) & $\begin{array}{l}0 / 0.40 \\
-\end{array}$ & $\begin{array}{l}0 / 0.28 \\
-\end{array}$ & $\begin{array}{l}0 / 0.06 \\
-\end{array}$ \\
\hline Chronic lymphoid leukaemia (204.1) & $\begin{array}{l}3 / 1.41 \\
-\end{array}$ & $\begin{array}{l}3 / 0.87 \\
-\end{array}$ & $0 / 0.33$ \\
\hline Acute myeloid (myelomonocytic) leukaemia (205.0) & $\begin{array}{l}1 / 1.27 \\
-\end{array}$ & $\begin{array}{l}1 / 0.84 \\
-\end{array}$ & $0 / 0.23$ \\
\hline Chronic myeloid leukaemia (205.1) & $\begin{array}{l}0 / 0.96 \\
-\end{array}$ & $\begin{array}{l}0 / 0.64 \\
-\end{array}$ & $\begin{array}{l}0 / 0.16 \\
-\end{array}$ \\
\hline $\begin{array}{l}\text { Other leukaemias }(204.2-204.9,205.2-205.9 \text {, } \\
206.1-206.9,207.2-207.8,208.0-208.9)\end{array}$ & $\begin{array}{l}0 / 0.55 \\
-\end{array}$ & $\begin{array}{l}0 / 0.35 \\
-\end{array}$ & $\begin{array}{l}0 / 0.09 \\
-\end{array}$ \\
\hline
\end{tabular}

*Expected malignancies based on Canadian general population cancer incidence rates. †95\% $\mathrm{Cl}$ does not include 1.00 .

$\mathrm{O} / \mathrm{E}$, observed/expected.

of the large intestine, the RR for the highest exposure group was slightly increased (RR 1.7 ) and not statistically significant.

\section{Petroleum coke/spent catalyst}

Only approximately $15 \%$ of the male cohort was exposed to petroleum coke/spent catalyst. There were insufficient numbers of exposed cases for most exposure-response analyses, although lung cancer showed 12 exposed cases. Less than half $(48.5 \%)$ of the male workers in the petroleum coke/spent catalyst cohort had known smoking histories. Since there were no cases classified as "never smoked", the models could not converge; thus, smoking was classified as ever/never (with unknown smokers classified as never smokers). Using this alternate smoking variable in the model, the risk of lung cancer was statistically significantly increased in the highest exposure group (RR 3.3; 95\% CI 1.4 to 7.7) but showed no clear exposure-response trend (see table 8). Results were similar without this smoking variable in the model.

We also examined SIRs by exposure category for petroleum coke/spent catalyst, as well as for the other substances where lung cancer was of a priori interest (that is, hydrocarbon 
Table 5 Distribution of exposure concentration estimates as an annual (2000 hour) average and number of individuals exposed by agent among Canadian petroleum workers, 1969-94

\begin{tabular}{|c|c|c|c|c|c|c|c|}
\hline \multirow[b]{2}{*}{ Agent } & \multicolumn{5}{|c|}{ Intensity estimates among exposed jobs } & \multirow{2}{*}{$\begin{array}{l}\text { No. of employees } \\
\text { in cohort }\end{array}$} & \multirow{2}{*}{$\begin{array}{l}\text { No. (\%) of cohor } \\
\text { exposed }\end{array}$} \\
\hline & 5th centile & 25 th centile & 50th centile & 75th centile & 95th centile & & \\
\hline $\begin{array}{l}\text { Petroleum coke/spent } \\
\text { catalyst }^{*}\end{array}$ & 0.03 & 0.25 & 3.00 & 20.00 & 84.00 & 25285 & $2804(11 \%)$ \\
\hline Hydrocarbon fuels $†$ & 0.01 & 0.30 & 1.77 & 6.85 & 47.98 & 25279 & $11717(46 \%)$ \\
\hline Hydrogen sulphide† & $<0.01$ & 0.01 & 0.07 & 0.60 & 4.76 & 25281 & $10577(42 \%)$ \\
\hline Hydrocarbon lubes* & 0.12 & 1.82 & 8.85 & 50.00 & 288.94 & 25285 & $9371(37 \%)$ \\
\hline
\end{tabular}

lubes and hydrocarbon solvents/fuels). Table 9 shows the results are consistent with expected for all exposure categories and all substances. Consistent with the RR results, there was a twofold increase in the highest exposure group for petroleum coke/spent catalyst.

\section{Hydrogen sulphide}

The $\mathrm{H}_{2} \mathrm{~S}$ analysis focused on non-malignant mortality given the lack of evidence to suggest a possible cancer risk associated with $\mathrm{H}_{2} \mathrm{~S}$. We examined respiratory disease mortality and "deaths due to accidental poisoning by other gases and vapours" because exposure to $\mathrm{H}_{2} \mathrm{~S}$ occurs by inhalation. Additionally, we assessed risk of death for neurological disorders, accidental falls, transportation accidents (with passengers removed from the analysis to the extent possible based on ICD codes), and suicide based on suggestions from several authors that low level, chronic exposure to $\mathrm{H}_{2} \mathrm{~S}$ may impair neuropsychological function. ${ }^{17-21}$

The results shown in table 10 indicate that deaths due to respiratory disease were non-significantly increased in the two exposure groups where the RR could be calculated, but these findings were based on small numbers. Suicide showed no relation to cumulative $\mathrm{H}_{2} \mathrm{~S}$ exposure. Finally, transportation accidents (for example, motor vehicle) showed an exposure-response trend, with risk in the highest cumulative exposure category reaching statistical significance (RR 2.3; $95 \%$ CI 1.2 to 4.4 ). There were too few deaths due to neurological disorders $(n=3)$, accidental falls, and accidental poisoning by other gases and vapours among exposed subjects for meaningful analyses.

\section{DISCUSSION}

This study examined mortality and cancer morbidity among a cohort of 25292 Canadian petroleum workers and explored exposure-response relations for four types of petroleum agents. Our study cohort has several advantages compared with previous cohorts from this Canadian petroleum company and other companies. First, by restricting the cohort to 1964 or later hires, healthy worker survivor bias is reduced and the results are more relevant to modern work environments. Second, only employees with complete ( $\geqslant 90 \%$ of work time assigned to SEGs) exposure histories were included. Finally, mortality information was supplemented with cancer incidence data, with the cancer incidence data being of high diagnostic quality (that is, $91 \%$ of the incident tumours were microscopically confirmed).

Interpretation of our findings must also consider several limitations. Because this was a cohort of relatively young employees with few deaths ( $2 \%$ deceased) and few incident tumours $(2 \%)$, statistical power is limited. While results account for age, time, and gender differences between the cohort and the national population, we could not account for other disease correlates related to lifestyle. We attempted to analyse information on smoking but only $43 \%$ of the cohort were classified as a known smoker or non-smoker, preventing adequate control for this important confounder. Another limitation is the large number of disease-exposure comparisons and other analyses (for example, tenure, latency) performed, which increases the likelihood of chance related findings. Also, case identification by the tumour registry may have been incomplete, especially in early time periods

Table 6 Rate ratios* and $95 \% \mathrm{Cl}$ by cumulative exposure to hydrocarbon solvents/fuels for selected $\dagger$ cancer incidence among males from a Canadian petroleum company, 1969-94

\begin{tabular}{|c|c|c|c|c|c|c|}
\hline $\begin{array}{l}\text { Hydrocarbon solvents/ } \\
\text { fuels (ppm-years) }\end{array}$ & $\begin{array}{l}\text { Malignant } \\
\text { melanoma (ICD } \\
\text { 9th revision 172) }\end{array}$ & $\begin{array}{l}\text { Bronchus \& lung } \\
\text { (ICD 9th revision } \\
162.2-5,162.8-9) \ddagger\end{array}$ & $\begin{array}{l}\text { Brain (malignant) } \\
\text { (ICD 9th revision } \\
\text { 191) }\end{array}$ & $\begin{array}{l}\text { Large intestine } \\
\text { (except rectum) } \\
\text { (ICD 9th revision } \\
153,159.0 \text { ) }\end{array}$ & $\begin{array}{l}\text { All lymphopoietic } \\
\text { cancers combined } \\
\text { (ICD 9th revision 200.0-2; } \\
200.8 ; 201 ; 202.0-1 ; \\
202.8-9 ; 203.0 ; 204-206 \text {; } \\
207.0,2,8 ; 208 \text { ) }\end{array}$ & $\begin{array}{l}\text { Non-Hodgkin's } \\
\text { lymphoma } \\
\text { (ICD 9th revision } \\
200.0-2,202.0 \text {, } \\
202.8 \text { ) }\end{array}$ \\
\hline $\begin{array}{l}\text { Unexposed } \\
>0-<2.5 \text { ppm-years } \\
\geqslant 2.5-<30.0 \text { ppm-years } \\
\geqslant 30 \text { ppm-years }\end{array}$ & $\begin{array}{l}1.0 \\
n=10 \\
1.1(0.4 \text { to } 3.0) \\
n=6 \\
1.2(0.4 \text { to } 3.4) \\
n=6 \\
0.6(0.2 \text { to } 2.1) \\
n=4\end{array}$ & $\begin{array}{l}1.0 \\
n=18 \\
0.8(0.3 \text { to } 1.8) \\
n=7 \\
0.7(0.3 \text { to } 1.8) \\
n=6 \\
1.2(0.6 \text { to } 2.4) \\
n=13\end{array}$ & $\begin{array}{l}1.0 \\
n=4 \\
0.9(0.2 \text { to } 5.1) \\
n=2 \\
2.7(0.7 \text { to } 10.0) \\
n=5 \\
2.0(0.5 \text { to } 7.4) \\
n=5\end{array}$ & $\begin{array}{l}1.0 \\
n=10 \\
1.9(0.8 \text { to } 4.6) \\
n=10 \\
1.1(0.4 \text { to } 3.2) \\
n=5 \\
1.5(0.6 \text { to } 3.9) \\
n=8\end{array}$ & $\begin{array}{l}1.0 \\
n=27 \\
1.3(0.7 \text { to } 2.3) \\
n=19 \\
0.7(0.3 \text { to } 1.4) \\
n=9 \\
0.3(0.1 \text { to } 0.8) \\
n=5\end{array}$ & $\begin{array}{l}1.0 \\
n=8 \\
2.1(0.8 \text { to } 5.4) \\
n=9 \\
0.3(0.1 \text { to } 1.3) \\
n=2 \\
\S \\
n=1\end{array}$ \\
\hline \multicolumn{7}{|c|}{$\begin{array}{l}\text { *All models include terms for age, time since hire, and calendar period. Number ( } \mathrm{n} \text { ) is number of cases in exposure category. } \\
\text { †Other malignancies evaluated but not shown due to less than } 10 \text { exposed cases includes kidney cancer (189.0-189.2), all leukaemias combined (204-206, } \\
207.0,207.2,207.8,208 \text { ) and Hodgkin's disease (201) (numbers in parentheses are ICD 9th revision codes). } \\
\ddagger \text { Model includes additional term for smoking (ever } v \text { never/unknown). } \\
\S \text { Exposure category combined with category above due to no or small number of cases. Rate ratio and confidence interval is based on observed counts in each cel } \\
\text { combined. The counts shown in each cell are the original counts before combined categories. }\end{array}$} \\
\hline
\end{tabular}


Table 7 Rate ratios* and $95 \% \mathrm{Cl}$ by cumulative exposure to hydrocarbon lubricants for selected $\dagger$ cancer incidence among males from a Canadian petroleum company, 1969-94

\begin{tabular}{|c|c|c|c|c|c|}
\hline $\begin{array}{l}\text { Hydrocarbon lubricants } \\
\text { (ppm-years) }\end{array}$ & $\begin{array}{l}\text { Malignant melanoma } \\
\text { (ICD 9th revision 172) }\end{array}$ & $\begin{array}{l}\text { Bronchus \& lung } \\
\text { (ICD 9th revision } \\
162.2-.5,162.8-.9) \ddagger\end{array}$ & $\begin{array}{l}\text { Brain (malignant) } \\
\text { (ICD 9th revision 191) }\end{array}$ & $\begin{array}{l}\text { Large intestine } \\
\text { (except rectum) } \\
\text { (ICD 9th revision } \\
153,159.0 \text { ) }\end{array}$ & $\begin{array}{l}\text { All lymphopoietic cancers } \\
\text { combined (ICD 9th revision } \\
200.0-2 ; 200.8 ; 201 ; \\
202.0-1 ; 202.8-9 ; 203.0 \text {; } \\
\text { 204-206; 207.0, 2, 8; 208) }\end{array}$ \\
\hline Unexposed & $\begin{array}{l}1.0 \\
n=13\end{array}$ & $\begin{array}{l}1.0 \\
n=21\end{array}$ & $\begin{array}{l}1.0 \\
n=6\end{array}$ & $\begin{array}{l}1.0 \\
n=15\end{array}$ & $\begin{array}{l}1.0 \\
n=33\end{array}$ \\
\hline$>0-<0.02$ ppm-years & $\begin{array}{l}1.2(0.4 \text { to } 3.0) \\
n=6\end{array}$ & $\begin{array}{l}1.2(0.5 \text { to } 2.5) \\
n=9\end{array}$ & $\begin{array}{l}1.3(0.3 \text { to } 5.1) \\
n=3\end{array}$ & $\begin{array}{l}1.1(0.4 \text { to } 2.8) \\
n=6\end{array}$ & $\begin{array}{l}1.1(0.6 \text { to } 2.1) \\
n=15\end{array}$ \\
\hline$\geqslant 0.02-<0.20$ ppm-years & $\begin{array}{l}0.9(0.4 \text { to } 2.2) \\
n=6\end{array}$ & $\begin{array}{l}0.8(0.3 \text { to } 2.0) \\
n=5\end{array}$ & $\begin{array}{l}1.6(0.4 \text { to } 6.4) \\
n=3\end{array}$ & $\begin{array}{l}1.3(0.5 \text { to } 3.4) \\
n=6\end{array}$ & $\begin{array}{l}0.8(0.4 \text { to } 1.7) \\
n=9\end{array}$ \\
\hline$\geqslant 0.20$ ppm-years & $\S$ & $\begin{array}{l}1.6(0.7 \text { to } 3.6) \\
n=9\end{array}$ & $\begin{array}{l}2.5(0.7 \text { to } 9.0) \\
n=4\end{array}$ & $\begin{array}{l}1.7(0.7 \text { to } 4.5) \\
n=6\end{array}$ & $\begin{array}{l}0.4(0.1 \text { to } 1.1) \\
n=3\end{array}$ \\
\hline
\end{tabular}

*All models include terms for age, time since hire, and calendar period. Number $(n)$ is number of cases in exposure category.

tOther malignancies evaluated but not shown due to less than 10 exposed cases includes kidney cancer (189.0-189.2), all leukaemias combined (204-206, 207.0, 207.2, 207.8, 208), Hodgkin's disease (201), and non-Hodgkin's lymphoma (200.0-2, 202.0, 202.8) (numbers in parentheses are ICD 9th revision codes).

$\ddagger$ Model includes additional term for smoking (ever $v$ never/unknown).

§Exposure category combined with category above due to no or small number of cases. Rate ratio and confidence interval is based on observed counts in each cell combined. The counts shown in each cell are the original counts before combined categories.

(1969-73), and in Quebec prior to 1977 (approximately 30\% in 1973/74). ${ }^{22}$ However, this is unlikely to have seriously biased the exposure specific results since unidentified incident case status is unlikely to be related to exposure.

There was a relatively high degree of correlation between exposures, especially hydrocarbon solvents/fuels and lubricants and $\mathrm{H}_{2} \mathrm{~S}$; this complicates interpretation of unique effects of these exposures. Some extrapolation of exposure estimates was necessary, and there may be heterogeneity within SEGs (for example, opportunity for peak exposures). While the above limitations are considerable, the strengths (for example, lack of survivor bias, relatively complete work history information) are such that the cohort's value will increase markedly in the future as the low power issue is lessened, especially for diseases that are more prevalent at younger ages.

\section{General mortality and cancer incidence}

The results suggest that employees have a favourable overall health profile. Deficits of deaths were observed for the major cause categories, which is consistent with other petroleum worker studies. ${ }^{10}{ }^{11}$ There also was a significant deficit in the incidence of all malignant neoplasms combined, a finding consistent with the few previous petroleum studies that have

Table 8 Rate ratios* and $95 \% \mathrm{Cl}$ by cumulative exposure to petroleum coke/spent catalyst for lung cancert incidence among males from a Canadian petroleum company, 1969-94

\begin{tabular}{ll}
\hline $\begin{array}{l}\text { Petroleum coke/spent catalyst } \\
\text { (mg/m } \mathbf{m}^{3} \text {-years) }\end{array}$ & $\begin{array}{l}\text { Bronchus \& lung (ICD 9th revision } \\
162.2-162.5,162.8-162.9)\end{array}$ \\
\hline Unexposed & 1.0 \\
& $\mathrm{n}=32$ \\
$>0-<0.005 \mathrm{mg} / \mathrm{m}^{3}$-years & $2.4(0.7$ to 7.8$)$ \\
& $\mathrm{n}=3$ \\
& $0.9(0.2$ to 3.9$)$ \\
& $\mathrm{n}=2$ \\
& $3.3(1.4$ to 7.7$) \ddagger$ \\
& $\mathrm{N}=7$ \\
\hline
\end{tabular}

*Model includes terms for age, time since hire, calendar period, and smoking. Number $(n)$ is number of cases in exposure category. tOther malignancies evaluated but not shown due to less than 10 exposed cases includes nose and sinus (160), larynx (161), bladder (188), and kidney cancer (189.0-189.2) (numbers in parentheses are ICD 9th revision codes).

$\$ 95 \% \mathrm{Cl}$ does not include value of 1.0 evaluated cancer incidence. ${ }^{23-26}$ It is reassuring that causes of death more common among younger age groups (for example, external causes, AIDS, suicide) and cancers with potentially shorter latencies (for example, LHC) were generally less than or consistent with expected. The reduced mortality and cancer incidence among these workers is likely a reflection of the healthy worker effect ${ }^{27}{ }^{28}$ and the lack of major occupational risks.

\section{Causes of a priori interest}

We found mesothelioma incidence to be increased compared with the general population. The number of cases was small $(\mathrm{n}=4)$, and two of the four cases had jobs with limited, if any, potential asbestos exposure. Unfortunately, we did not have data on potential asbestos exposures prior to employment with the company. However, there appeared to be higher risk for long term, long latency workers, which is in agreement with our previous update ${ }^{1}$ and with other petroleum worker studies. ${ }^{29-36}$ Given that the cohort included only employees hired in 1964 or later, the findings suggest the need for continued attention to asbestos in the petroleum industry.

Unlike some petroleum worker studies, ${ }^{9}$ we found no evidence of an increased risk of overall leukaemia, lymphoma, or related malignancies. Our limited findings are consistent with a meta-analysis of cell type specific leukaemia mortality among over 208000 petroleum workers in the USA and UK, ${ }^{37}$ and a case-control study of benzene and LHC nested within an earlier cohort of these Canadian workers. ${ }^{38}$

We also found no evidence of an increased risk of kidney cancer. While there are some epidemiology $y^{39-41}$ and toxicology $^{42}$ data to suggest an association with petroleum exposures, our findings are consistent with several studies that have found no relation between kidney cancer and hydrocarbons in refinery, ${ }^{43}{ }^{44}$ marketing, ${ }^{45}$ and petrochemical ${ }^{46}$ workers.

While the overall SMR and SIR for large intestine and brain cancer were unremarkable, the large intestine cancer SIR showed a trend with employment duration. Both large intestine and brain cancer showed a weakly suggestive exposure-response trend with hydrocarbon lubricants. Previous studies of workers from this Canadian petroleum company have found large intestine cancer to be increased, ${ }^{2}$ particularly among marketing/distribution workers (some of whom were in this cohort) where an inconsistent association with total hydrocarbon exposure has been observed. ${ }^{3}$ In another petroleum distribution worker study in the UK, an 


\begin{tabular}{|c|c|c|c|}
\hline Exposure category $\dagger$ & $\begin{array}{l}\text { Petroleum coke/spent } \\
\text { catalyst } \\
\text { SIR }(95 \% \mathrm{CI}) \\
\text { Observed }\end{array}$ & $\begin{array}{l}\text { Hydrocarbon lubricants } \\
\text { SIR (95\% CI) } \\
\text { Observed }\end{array}$ & $\begin{array}{l}\text { Hydrocarbon solvents/ } \\
\text { fuels } \\
\text { SIR }(95 \% \mathrm{CI}) \\
\text { Observed }\end{array}$ \\
\hline Unexposed & $\begin{array}{l}\mathrm{SIR}=0.58 \\
(0.40 \text { to } 0.82) \\
\text { Obs }=32\end{array}$ & $\begin{array}{l}\mathrm{SIR}=0.63 \\
(0.39 \text { to } 0.96) \\
\text { Obs }=21\end{array}$ & $\begin{array}{l}\text { SIR }=0.72 \\
(0.43 \text { to } 1.14) \\
\text { Obs }=18\end{array}$ \\
\hline Lowest exposure tertile & - & $\begin{array}{l}\mathrm{SIR}=0.71 \\
(0.33 \text { to } 1.36)\end{array}$ & $\begin{array}{l}\mathrm{SIR}=0.54 \\
(0.22 \text { to } 1.11)\end{array}$ \\
\hline & $\mathrm{Obs}=3$ & $\mathrm{Obs}=9$ & Obs $=7$ \\
\hline Middle exposure tertile & - & $\begin{array}{l}\mathrm{SIR}=0.50 \\
(0.16 \text { to } 1.16)\end{array}$ & $\begin{array}{l}\mathrm{SIR}=0.52 \\
(0.19 \text { to } 1.14)\end{array}$ \\
\hline Highest exposure tertile & $\begin{array}{l}\text { Obs }=2 \\
\text { SIR }=2.17 \\
(0.87 \text { to } 4.48) \\
\text { Obs }=7\end{array}$ & $\begin{array}{l}\text { Obs }=5 \\
\text { SIR }=1.13 \\
(0.52 \text { to } 2.15) \\
\text { Obs }=9\end{array}$ & $\begin{array}{l}\text { Obs }=6 \\
\text { SIR }=0.89 \\
(0.48 \text { to } 1.53) \\
\text { Obs }=13\end{array}$ \\
\hline
\end{tabular}

*Expected numbers based on cancer incidence rates for the general Canadian population. †Units for exposure tertiles varies by substance (see tables 6-8 for units).

increase in large intestine cancer was focused among office and clerical workers. ${ }^{47}$ Moreover, a large study of workers exposed to lubricants during metal working and machining reported an exposure-response relation for rectal but not large intestine cancer, ${ }^{48}$ but another similar study found no relation for either of these malignancies. ${ }^{49}$ Finally, the plausibility of an inhalation related exposure to lubricants targeting the large intestine is limited.

Several petroleum worker studies have reported increased deaths due to brain cancer, ${ }^{35}{ }^{36}$ 50-53 $^{5}$ with some providing a weak suggestion of an association with lubricant exposure. $^{5253}$ However, two large studies of metal working employees have found no evidence of increased brain cancer. ${ }^{48}{ }^{49}$ Overall, our results provide only weak evidence, at best, of a possible risk for large intestine and brain cancer. Nevertheless, it will be important to monitor these diseases in future updates.

Finally, malignant melanoma mortality was increased in earlier studies from this company, especially among upstream workers. ${ }^{1-3}$ SIR results showed slight increases, while substance specific analyses showed no evidence of an association. Our results are consistent with most other petroleum worker studies, which have not found skin cancer and/or malignant melanoma to be increased..$^{10}$

\section{Other health outcomes \\ Lung cancer}

While there were deficits of lung cancer mortality and morbidity among employees compared with the general population, the Poisson regression analyses showed an irregular exposure-response pattern for petroleum coke/spent catalyst exposure, with a statistically significant threefold RR of lung cancer among workers in the highest cumulative exposure category. The SIR analyses showed a similar, but smaller and non-significant increase in the highest cumulative petroleum coke/spent catalyst exposure group. We were not able to adequately account for smoking because there were no employees classified as non-smokers among the lung cancer cases. We also did not have data on socioeconomic

Table 10 Rate ratios ${ }^{*}$ and $95 \% \mathrm{Cl}$ by cumulative exposure to hydrogen sulphide $\left(\mathrm{H}_{2} \mathrm{~S}\right)$ for selected non-malignant causes of death among males from a Canadian petroleum company, 1964-94

\begin{tabular}{|c|c|c|c|}
\hline $\begin{array}{l}\text { Hydrogen sulphide } \\
\text { (ppm-years) }\end{array}$ & $\begin{array}{l}\text { Non-malignant } \\
\text { respiratory disease } \\
\text { (ICD 9th revision } \\
460-519 \text { ) }\end{array}$ & $\begin{array}{l}\text { Suicide (ICD 9th revision } \\
\text { E950-E959) }\end{array}$ & $\begin{array}{l}\text { Transportation } \\
\text { accidents§ }\end{array}$ \\
\hline & $\begin{array}{l}1.0 \\
n=5\end{array}$ & $\begin{array}{l}1.0 \\
n=19\end{array}$ & $\begin{array}{l}1.0 \\
n=23\end{array}$ \\
\hline$>0-<0.1$ ppm-years & $\begin{array}{l}1.8(0.5 \text { to } 6.8) \\
n=4\end{array}$ & $\begin{array}{l}1.3(0.6 \text { to } 2.7) \\
n=12\end{array}$ & $\begin{array}{l}1.2(0.6 \text { to } 2.3) \\
n=13\end{array}$ \\
\hline$\geqslant 0.1-<1.5$ ppm-years & $\begin{array}{l}2.2(0.7 \text { to } 7.3) \\
n=6\end{array}$ & $\begin{array}{l}1.0(0.4 \text { to } 2.3) \\
\mathrm{n}=8\end{array}$ & $\begin{array}{l}1.8(0.9 \text { to } 3.3) \\
n=17\end{array}$ \\
\hline$\geqslant 1.5$ ppm-years & $n=0$ & $\begin{array}{l}0.9(0.3 \text { to } 2.3) \\
n=5\end{array}$ & $\begin{array}{l}2.3(1.2 \text { to } 4.4)^{* *} \\
n=15\end{array}$ \\
\hline
\end{tabular}

*All models include terms for age and calendar period. Number $(n)$ is number of cases in exposure category. tOther causes of death evaluated but not shown due to less than 10 exposed cases includes neurological disorders (320-389), deaths due to accidental poisoning by other gases and vapours (E869.8) and accidental falls (E880E888) (numbers in parentheses are ICD 9th revision codes).

$\ddagger$ Model includes additional term for smoking (ever $v$ never/unknown).

$\S$ Transportation accidents based on 9th revision ICD codes for motor vehicle accidents (E810-E819), motor vehicle non-traffic accidents (E820-E825), other road vehicle accidents (E826-E829), water transport accidents (E830E838), and air \& space transport accidents (E840-E845). Passengers were excluded from the analysis to the extent possible based on the 4 th digit of the ICD code when specified.

- Exposure category combined with category above due to no or small number of cases. Rate ratio and confidence interval is based on observed counts in each cell combined. The counts shown in each cell are the original counts before combined categories.

**95\% Cl does not include value of 1.0 . 
status, which could also explain a higher lung cancer risk for the higher exposed workers.

Carbon is the primary (84-97\%) component in petroleum coke, although polycyclic aromatic hydrocarbons (PAH) and metals (that is, vanadium) also may be present, depending on the type of production process (the production unit in this study was a fluidised catalytic cracking unit). ${ }^{54}$ The low level of exposure in our cohort (see table 5) detracts from the plausibility of the observed threefold increased risk, although it is possible that peak exposures may have occurred which were not fully represented in our exposure estimates. Finally, spent catalyst dusts contain metallic agents (for example, nickel, palladium), and some metallic agents have been shown to be carcinogenic to the human lung (for example, arsenic, chromium). ${ }^{55}$ However, we did not have data on the type, level, and composition of spent catalyst exposure.

The available toxicology data for petroleum coke apply most directly to delayed and fluid coke and indicate little evidence of carcinogenic risk..$^{56}$ The product of the delayed coking process, delayed (green) coke, contains significant levels of volatile hydrocarbons, including PAHs. Fluid coke is the product of a continuous fluidised bed coking process and is similar in composition to delayed coke. Mutagenicity tests for delayed and fluid coke have been negative..$^{57-59}$

Previously published studies of petroleum workers have observed consistent, significant deficits of lung cancer mortality compared with the general population, ${ }^{10}{ }^{11}$ although none have examined mortality by exposure to petroleum coke. The only epidemiology study we are aware of to report on petroleum coke workers is Divine and colleagues, ${ }^{60}$ who found a deficit of lung cancer deaths among petroleum workers ever employed (SMR 0.65; 95\% CI 0.47 to 0.87 , $\mathrm{O}=44$ ) or employed five or more years (SMR $0.84 ; 95 \% \mathrm{CI}$ 0.43 to $1.47, \mathrm{O}=12$ ) in delayed coke processing between 1947 and 1993.

In summary, the petroleum coke/spent catalyst and lung cancer association is statistically significant in the highest exposure group, has some biological plausibility (for example, PAHs, metallic agents), and was of a priori interest. As such, the findings could be argued to be suggestive of a possible occupational relation. However, the existence of only one other (negative) study, the small number of exposed lung cancer cases, the irregular exposure-response, and the inability to adequately account for the potentially large confounding effects of smoking argues against an association. It is possible that additional studies with a greater number of cases, improved data on smoking, and better characterisation of these and other potential exposures may help clarify these findings.

\section{Gall bladder cancer}

While gall bladder cancer showed a statistically significantly increased SIR among males, this cause was not of a priori interest, there was no common job assignment among cases, and the SIR showed no trend with duration of employment or time since hire. Only one previous study of refinery workers reported results for gall bladder cancer (SMR 0.95; $95 \%$ CI 0.70 to $1.26, \mathrm{n}=47$ deaths) ${ }^{41}$ On balance, our findings for gall bladder cancer are most likely due to chance, given the large number of comparisons made.

\section{Transportation accidents}

Our results indicate a statistically significant RR for deaths due to transportation accidents in the highest cumulative exposure category, with evidence of an exposure-response trend. Several authors of experimental studies in animals ${ }^{17-19}$ and/or case reports or community surveys in humans s $^{21} 61$ have interpreted their findings to be consistent with a neurological effect from low level $\mathrm{H}_{2} \mathrm{~S}$ exposure. There have also been reports that repeated or prolonged "knockdowns" (that is, loss of consciousness) from high level $\mathrm{H}_{2} \mathrm{~S}$ exposure result in prolonged adverse neurological effects, ${ }^{62-64}$ although evidence in this area remains highly controversial. ${ }^{63}$ We did not have data on peak exposures or "knockdown" events to investigate the role of intermittent, high exposures.

The final interpretation of our results is complicated by several potential confounding factors that we could not account for (for example, duration of time spent in vehicles, which may vary depending on rural versus urban locations, shift work, etc). As this is the first study to our knowledge to examine risk of accidental death associated with chronic $\mathrm{H}_{2} \mathrm{~S}$ exposure, no strong inferences can be made without investigation in other populations.

\section{Summary and conclusion}

The results indicated a favourable overall health profile for the cohort, with deaths due to all causes and all malignant neoplasms combined and many specific causes being less than expected. Cancer incidence findings were similar, although mesothelioma was increased in long duration/ latency employees. Exposure specific analyses suggested an exposure-response trend for cumulative $\mathrm{H}_{2} \mathrm{~S}$ exposure and transportation accidents. However, several unmeasured confounders exist, and there are no similar studies for comparison. As such, the $\mathrm{H}_{2} \mathrm{~S}$ findings need to be verified through studies of other similarly exposed workers. We also observed an increased risk of lung cancer among workers in the highest cumulative exposure category for petroleum coke/ spent catalyst; but this analysis could not adequately control for smoking, was based on small numbers, exhibited a tenuous exposure-response pattern, and is the first study to report this association. It is possible that additional studies with a greater number of cases, improved data on smoking, and better characterisation of these and other potential exposures may help clarify the findings.

\section{ACKNOWLEDGEMENTS}

The authors gratefully acknowledge the contributions of Martha Fair, Pierre Lalonde, and Dores Zuccarini in the Occupational and Environmental Health Research Section (OEHRS), Health Statistics Division of Statistics Canada, and the Provincial and Territorial Vital Statistics and Cancer Registries. The authors would also like to thank Eugene Sales for nosological coding, Celia Milano for programming, Shirin Sotoudeh and Gail Wilson for data preparation, Wendy Huebner for comments on the manuscript, and Tom Armstrong for guidance in agent selection and exposure estimating oversight. Sponsor: Imperial Oil Limited.

\section{Authors' affiliations}

R J Lewis, A R Schnatter, G Jorgensen, M J Nicolich, ExxonMobil Biomedical Sciences, Inc., 1545 Route 22, PO Box 971, Room LF 264, Annandale, NJ 08801-0971, USA

I Drummond, N Murray, F S Thompson, A M Katz, D Dahlman, Imperial Oil Limited, 111 St Clair Ave, Toronto, Ontario, Canada M5W 1K3

G Thériault, McGill University, Joint Dept of Epidemiology \& Biostatistics, and Occupational Health, 1020 Pine Avenue West, Montreal, Quebec, Canada H3A 1A2

\section{REFERENCES}

1 Lewis RJ, Schnatter AR, Katz AM, et al. Updated mortality among diverse operating segments of a petroleum company. Occup Environ Med 2000:57:595-604.

2 Schnatter AR, Theriault G, Katz AM, et al. A retrospective mortality study within operating segments of a petroleum company. Am J Ind Med 1992;22:209-29.

3 Schnatter AR, Katz AM, Nicolich MJ, et al. A retrospective mortality study among Canadian petroleum marketing and distribution workers. Environ Health Perspect 1993;101:85-99.

4 Chemical Manufacturers Association Epidemiology Task Group. Guidelines for good epidemiology practices for occupational and environmental epidemiologic research. J Occup Med 1991;33:1221-9. 
5 Drummond I, Murray N, Armstrong TW, et al. Exposure assessment methods for a study of mortality and cancer morbidity in relation to specific petroleum industry exposures. Unpublished report, 2002.

6 International Agency for Research on Cancer (IARC), International Association of Cancer Registries. Multiple primaries. IARC International Report No. 94/003 Lyon, 1994.

7 Cunningham J, Hamkey B, Lyles B, et al. SEER program code manual, revised edn. Cancer Statistics Branch, National Cancer Institute, 1992

8 Hanis NM, Stavraky KM, Fowler JL. Cancer mortality in oil refinery workers. J Occup Med 1979:21:167-74.

9 International Agency for Research on Cancer (IARC). Monograph on the evaluation of carcinogenic risks to humans: occupational exposures in petroleum refining, crude oil, and major petroleum fuels. IARC Monograph 1989:45:39-117.

10 Delzell E, Austin H, Cole P. Epidemiologic studies of the petroleum industry. Occup Med 1988;3:455-74

11 Wong O, Raabe GK. Critical review of cancer epidemiology in petroleum industry employees, with a quantitative meta-analysis by cancer site. Am J Ind Med 1989:15:283-310.

12 Schnatter AR, Acquavella JF, Thompson FS, et al. An analysis of death ascertainment and follow-up through Statistics Canada's mortality data base system. Can J Public Health 1990;81:60-5.

13 Acquavella JF, Donaleski D, Hanis NM. An analysis of mortality follow-up through the national death index for a cohort of refinery and petrochemical workers. Am J Ind Med 1986;9:181-7.

14 Marsh GM, Ehland J, Park M, et al. OCMAP/PC: a user-oriented cohort mortality analysis program for the IBM PC. Am Stat 1986:40:308-9.

15 Haenszel W, Loveland D, Sirkin MG. Lung cancer mortality as related to residence and smoking histories. J Natl Cancer Inst 1962;28:947-1001.

16 SAS Institute Inc. SAS Technical Report P-243. SAS/STAT Software: The GENMOD procedure, release 6.09. Cary, NC: SAS Institute Inc., 1993.

17 Roth SH, Skrainy B, Reiffenstein RJ. Alteration of the morphology and neurochemistry of the developing mammalian nervous system by hydrogen sulphide. Clin Exp Pharmacol Physiol 1995;22:379-80

18 Roth SH. Toxicological effects of hydrogen sulfide in mammalian brain Environ Epidemiol Toxicol 1999;1:241-8.

19 Kilburn KH. Function testing for chemical brain damage: a review. Arch Environ Health 2001;56:132-7.

20 United States Environmental Protection Agency. Health assessment document for hydrogen sulfide. EPA/600/8-86/026F. Washington, DC: Office of Research and Development, 1993.

21 Legator MS, Singleton CR, Morris DL, et al. Health effects from chronic low level exposure to hydrogen sulfide. Arch Environ Health 2001;56:123-31.

22 Canadian Council of Cancer Registries, Health and Welfare Canada, Statistics Canada. The making of the Canadian Cancer Registry: cancer incidence in Canada and its regions, 1969 to 1988. Ottawa, 1993.

23 Christie D, Robinson K, Gordon I, et al. A prospective study in the Australian petroleum industry. II. Incidence of cancer. Br J Ind Med 1991;48:511-14.

24 Jarvholm B, Mellblom B, Norrman R, et al. Cancer incidence of workers in the Swedish petroleum. Occup Environ Med 1997;54:686-91.

25 Pukkala E. Cancer incidence among Finnish oil refinery workers, 1971-1994. J Occup Environ Med 1998;40:675-9.

26 Huebner WW, Chen WW, Friedlander BR, et al. Incidence of lymphohaematopoietic malignancies in a petrochemical industry cohort: 1983-94 follow up. Occup Environ Med 2000;57:605-14.

27 Fox AJ, Collier PF. Low mortality rates in industrial cohort studies due to selection for work and survival in the industry. Br J Prev Soc Med 1976;30:225-30.

28 Monson RR. Observations on the healthy worker effect. J Occup Med 1986:28:425-33.

29 Gennaro V, Ceppi M, Boffetta P, et al. Pleural mesothelioma and asbestos among Italian oil refinery workers. Scand J Work Environ Health 1994;20:213-15

30 Kaplan SD. Update of a mortality study of workers in petroleum refineries. J Occup Med 1986;28:514-16.

31 Tsai SP, Waddell LC, Gilstrap EL, et al. Mortality among maintenance employees potentially exposed to asbestos in a refinery and petrochemical plant. Am J Ind Med 1996;29:89-98.

32 Honda $Y$, Delzell E, Cole P. An updated study of morality among workers at a petroleum manufacturing plant. J Occup Environ Med 1995;37:194-200.

33 Huebner WW, Schnatter AR, Nicolich MJ, et al. Mortality experience of a young petrochemical industry cohort: 1979-1992 follow-up study of USbased employees. J Occup Environ Med 1997;39:970-82.

34 Satin KD, Wong O, Yuan LA, et al. A 50-year mortality follow-up of a large cohort of oil refinery workers in Texas. J Occup Environ Med 1996;38:492-506.
35 Tsai SP, Gilstrap EL, Colangelo TA, et al. A mortality study of oil refinery and petrochemical employees. J Occup Environ Med 1997;39:448-54.

36 Divine BJ, Hartman CM, Wendt JK. Update of the Texaco mortality study 1947-93: Part II. Analysis of specific causes of death for white men employed in refining, research, and petrochemicals. Occup Environ Med 1999;56:174-80

37 Wong O, Raabe GK. Cell-type-specific leukemia analyses in a combined cohort of more than 208,000 petroleum workers in the United States and the United Kingdom, 1937-1989. Regul Toxicol Pharmacol 1995;21:307-21.

38 Schnatter AR, Armstrong TW, Nicolich MJ, et al. Lymphohaematopoietic malignancies and quantitative estimates of exposure to benzene in Canadian petroleum distribution workers. Occup Environ Med 1996;53:773-81.

39 Shallenberger LG, Acquavella JF, Donaleski D. An updated mortality study of workers in three major United States refineries and chemical plants. $\mathrm{Br} J$ Ind Med 1992;49:345-54.

40 Siemiatycki J, Dewar R, Nadon L, et al. Associations between several sites of cancer and twelve petroleum-derived liquids. Results from a case-referent study in Montreal. Scand J Work Environ Health 1987;13:486-92.

41 Rushton L. Further follow-up of mortality in a United Kingdom oil refinery cohort. Br J Ind Med 1993:50:549-60.

42 MacFarland HN, Ulrich CE, Holdsworth CE, et al. A chronic inhalation study with unleaded gasoline vapor. J Am Coll Toxicol 1984;1984:231-48.

43 Poole C, Dreyer NA, Satterfield MH, et al. Kidney cancer and hydrocarbon exposures among petroleum refinery workers. Environ Health Perspect 1993;101(suppl 6):53-62.

44 Wen CP. Epidemiological studies of the role of gasoline (hydrocarbon) exposure in kidney cancer risk. Adv Mod Environ Toxicol 1984;7:245-58.

45 Wong O, Harris F, Smith TJ. Health effects of gasoline exposure. II. Mortality patterns of distribution workers in the United States. Environ Health Perspect 1993;101(suppl 6):63-76.

46 Gamble JF, Pearlman ED, Nicolich MJ. A nested case-control study of kidney cancer among refinery/petrochemical workers. Environ Health Perspect 1996; 104:642-50.

47 Rushton L. Further follow-up of mortality in a United Kingdom oil distribution centre cohort. Br J Ind Med 1993;50:561-9.

48 Tolbert PE, Eisen EA, Pothier $\amalg$, et al. Mortality studies of machining-fluid exposure in the automotive industry II. Risks associated with specific fluid types. Scand J Work Environ Health 1992;18:351-60.

49 Kazerouni N, Thomas TL, Petralia SA, et al. Mortality among workers exposed to cutting oil mists: update of previous reports. Am J Ind Med 2000;38:410-16.

50 Consonni D, Pesatori AC, Tironi A, et al. Mortality study in an Italian oil refinery: extension of the follow-up. Am J Ind Med 1999;35:287-94.

51 Theriault G, Provencher S. Mortality study of oil refinery workers: five-year follow-up. J Occup Med 1987;29:357-60.

52 Divine BJ, Hartman CM, Wendt JK. Update of the Texaco mortality study 1947-93: Part I. Analysis of overall patterns of mortality among refining, research, and petrochemical workers. Occup Environ Med 1999;56:167-73.

53 Thomas TL, Stewart PA, Stemhagen A, et al. Risk of astrocytic brain tumors associated with occupational chemical exposures. Scand J Work Environ Health 1987:13:417-23.

54 CONCAWE. Petroleum coke. Product Dossier No. 93/105. 1993

55 Shottenfeld D, Fraumeni Jr JF. Cancers of the lung and pleura. In: Shottenfeld D, Fraumeni Jr JF, eds. Cancer epidemiology and prevention, 2nd edn. New York: Oxford University Press, 1996.

56 Klonne DR, Burns JM, Halder CA, et al. Two-year inhalation toxicity study of petroleum coke in rats and monkeys. Am J Ind Med 1987;1 1:375-89.

57 Monarca S, Pasquini R, Sforzolini GS, et al. Application of the salmonella mutagenicity assay and determination of polycyclic aromatic hydrocarbons in workplaces exposed to petroleum pitch and petroleum coke. Int Arch Occup Environ Health 1982;49:223-39.

58 American Petroleum Institute. In vivo and in vitro mutagenicity studiesdelayed process coke. A revised final report. API 28-30728, 20 January, 1981

59 American Petroleum Institute. In vivo and in vitro mutagenicity studies - fluid process coke. A revised final report. API 28-30726, 20 January 1981

60 Divine BJ, Hartman CM, Wendt JK. An updated report on the Texaco mortality study 1947-1993. Internal report, October 1996.

61 Milby TH, Baselt RC. Hydrogen sulfide poisoning: clarification of some controversial issues. Am J Ind Med 1999:35:192-5.

62 Guidotti TL. Hydrogen sulphide. Occup Med 1996;46:367-71.

63 Guidotti TL. Occupational exposure to hydrogen sulfide in the sour gas industry: some unresolved issues. Int Arch Occup Environ Health 1994:66:153-60

64 Hessel PA, Melenka LS. Health effects of acute hydrogen sulfide exposures in oil and gas workers. Environ Epidemiol Toxicol 1999;1:201-6. 